\title{
Integrating Berth Allocation Decisions in a Fleet Composition and Periodic Routing Problem of Platform Supply Vessels
}

\author{
Roberto Cruz ${ }^{\mathrm{a}, \mathrm{c}, *}$, André Bergsten Mendes ${ }^{\mathrm{a}}$, Laura Bahiense ${ }^{\mathrm{b}}$, Yue $\mathrm{Wu}^{\mathrm{d}}$ \\ ${ }^{a}$ Department of Naval Architecture and Ocean Engineering, University of Sao Paulo, Sao \\ Paulo, Brazil \\ ${ }^{b}$ Systems Engineering and Computer Science Program, Transportation Engineering \\ Program, Alberto Luiz Coimbra Institute - Graduate School and Research in Engineering, \\ Federal University of Rio de Janeiro, Rio de Janeiro, Brazil \\ ${ }^{c}$ CENPES, Petrobras RED Center, Rio de Janeiro, Brazil \\ ${ }^{d}$ Southampton Business School, University of Southampton, Southampton, UK.
}

\begin{abstract}
The aim of this work is to present mathematical models and a heuristic solution strategy to solve the heterogeneous fleet-sizing problem of platform supply vessels (PSVs) that support the offshore oil and gas exploration and production $(\mathrm{E} \& \mathrm{P})$ activities. The problem considered in this research takes into account a novel characteristic related to the berth allocation problem of the supply base, which must be considered together with the decisions of selecting the departure days and the routes. The adopted solution strategy consists of sequentially solving models that capture different aspects of the problem, by starting with models that are simpler to solve. The solution found in one step provides a lower bound to the next step. This procedure was devised in order to reduce the search space and to speed up convergence. The proposed solution strategy was applied to real instances in Brazil, which has up to 79 offshore units grouped into clusters, with fair/acceptable results. The procedure allowed for assessing the impact of the number of berths on the fleet composition.
\end{abstract}

*Corresponding author. Tel.: (+55) 11 3091-5689.

Email addresses: redwacruz@yahoo.com.br (Roberto Cruz), andbergs@usp.br (André Bergsten Mendes), laura@cos.ufrj.br (Laura Bahiense), y.wu@soton.ac.uk (Yue $\mathrm{Wu}$ ) 
Keywords: OR in Maritime Industry, Heterogeneous Fleet Sizing, Berth Allocation, Periodic Routing, Offshore Supply Vessels

\section{Introduction}

Oil and gas offshore exploration and production (E\&P) has been facing the challenge of coping with the oil price crisis that has affected the entire segment worldwide since 2015. Particularly in the offshore support vessel market, the declining oil prices appear to be having a negative and worrying impact. Support vessels' service providers have not only to deal with the lower freight rates for the time charter and spot markets, but also with the segment over-capacity. Fortunately, the existing offshore oilfields have maintained their operating levels, and the demand for support vessels in ongoing operations has not suffered major impacts.

Irrespective of the crisis and its impact on the offshore support vessels' market, E\&P operators face continuous pressure to reduce costs. Even though operational expenditure (OPEX) is not as high when compared to capital expenditure (CAPEX) for offshore E\&P, there is always room for the pursuit of reductions in operational costs. In the upstream segment of the oil and gas supply chain, apart from the costs related to operations and interventions in wells and subsea systems, the main operational cost is associated with the platform supply vessels (Halvorsen-Weare and Fagerholt, 2017). These vessels play an important role in an oilfield development as they are present throughout the whole of the offshore E\&P life cycle (exploration, production and demobilization). The impact of reducing their costs seems to be quite relevant when considering that fields some distance from shore have relatively high density in terms of number of offshore units to be serviced.

The role of the platform supply vessels (PSV) in the logistics of the offshore oil industry is described in Aas et al. (2009); it consists of delivering goods from an onshore supply base to one or more offshore units, and returning items from these units to the onshore base. A PSV usually has the capacity to carry both bulk cargo and deck cargo. While the former - consisting of water, diesel and special fluids - is stored in tanks under the main deck, the latter consists of general cargo that is stored in offshore containers which are lifted by cranes from the vessels' main decks. In most cases the E\&P operators charter the fleet of PSVs rather than owning them. The hiring rates are highly dependent on the contract duration - long term (time 
charter) or short term (spot) - and on the deck area capacity, as described in Døsen and Langeland (2015).

From the E\&P operators' perspective, or even from the perspective of offshore logistics providers, cost savings could be achieved through a better planning of the PSV fleet. In the problem considered in this paper, the fleet planning must also take into consideration the berth capacity constraints in a $24 \times 7$ continuous operation, being the berth time dependent on the amount of cargo loaded rather than being a fixed time. This paper proposes a mathematical model for the fleet composition and periodic routing problem of platform supply vessels integrated with the berth allocation problem. We also propose a solution strategy to solve the mathematical model in steps, which was devised to reduce the processing time. Lastly, real instances from a Brazilian oil and gas company are solved. This article has the following structure: problem description, literature review, mathematical model, solution strategy and the case study with the computational results, followed by the concluding remarks and suggestions for future work.

\section{Problem Description}

In order to properly introduce the problem, the most important characteristics are presented first: i) Regular weekly schedules must be established to serve the offshore units. This is the current praxis and brings the required discipline for all agents involved in the process - suppliers, supply base operators, cargo planners and clients. The schedules are reviewed from time to time mostly due to location changes of mobile units or due to special operations (e.g., installation campaigns, decommissioning campaigns, or planned maintenance). Figure 1 shows an example of the routes servicing 15 offshore units. As in the work of Kisialiou et al. (2018), the one-week period is called 'the planning horizon of the installations'. As for the vessels, their schedule must fit a two-week period, known as 'the planning horizon of the vessels'. By considering a longer planning horizon for the vessels, trips may begin by the end of the first week, giving more flexibility to the route-planning process. Therefore, one vessel might not repeat the same route in the next evaluation period. If the planning horizon for the vessels was limited to one period, the routes that starts in the first period and finish in the second period would never be feasible, as exemplified by the black route that departs on day 6 in Figure 2. ii) The frequency of visits requested by an offshore unit within a week is known and depends on several factors. Usually the production units 
have a more stable demand with lower fluctuations and, as a consequence, the demand is more predictable. In this case, a lower frequency is required. On the other hand, demand is more unpredictable for drilling rigs due to unforeseen problems that often occur. Therefore, for drilling rigs and for some special units that work in campaigns, e.g., maintenance units, higher frequencies of visit are imposed. iii) The vessels' departure days to serve an offshore unit should be evenly spread as much as possible. This approach is the same used in the works of Halvorsen-Weare and Fagerholt (2017) and Halvorsen-Weare et al. (2012). An example of departure patterns from the supply base for frequencies of one-, two- and three-weekly visits can be seen in Figure 3. iv) Offshore units are grouped into clusters. A cluster consists of a group of nearby platforms that are served together, as a separate entity from the others. They belong to the same oilfield and are operated by the same onshore base. This segregation is observed in the Brazilian offshore $\mathrm{E} \& \mathrm{P}$, and allows a better management of the logistics process, including cargo prioritization and solving disputes more easily. Figure 4 presents a set of 79 offshore units grouped into nine clusters. Each cluster is indicated by a different color and each dot represents an offshore unit. Even though platforms are grouped into clusters, the frequency of visits is defined individually for each platform.

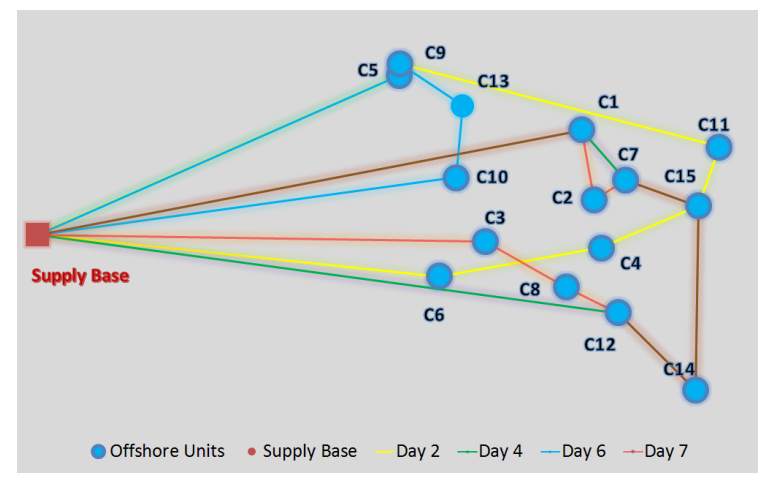

Figure 1: Example of departures routes per day for 15 offshore units.

The problem is also characterized by the following: v) The PSV fleet is categorized by the vessels' deck capacity. In this research three classes of PSV are being considered. The first one is PSV4500 with deck capacity of up to $900 \mathrm{~m}^{2}$, the second one is PSV3000 with deck capacity of up to $600 \mathrm{~m}^{2}$, and the third is PSV1500 with deck capacity of up to $300 \mathrm{~m}^{2}$. vi) The PSVs 


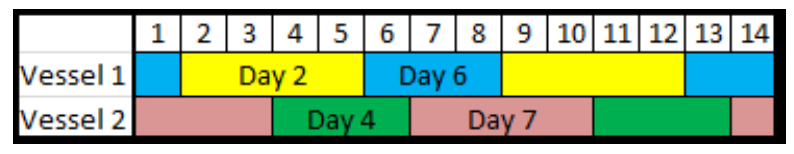

Figure 2: Example of vessels' allocation per day for 15 offshore units.

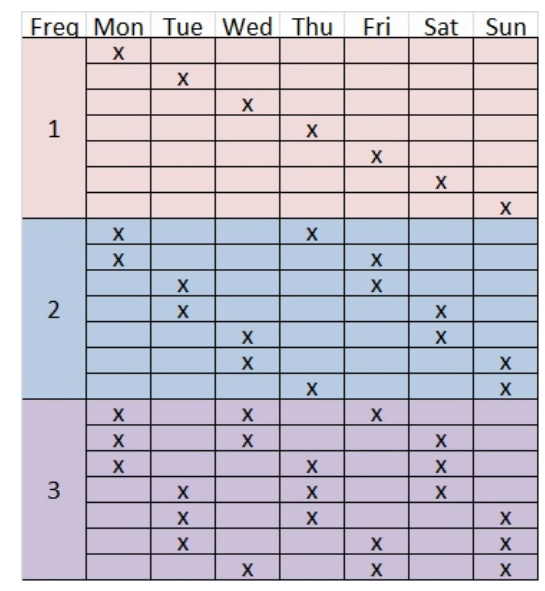

Figure 3: Departure patterns for frequencies of one-, two- and three-weekly visits.

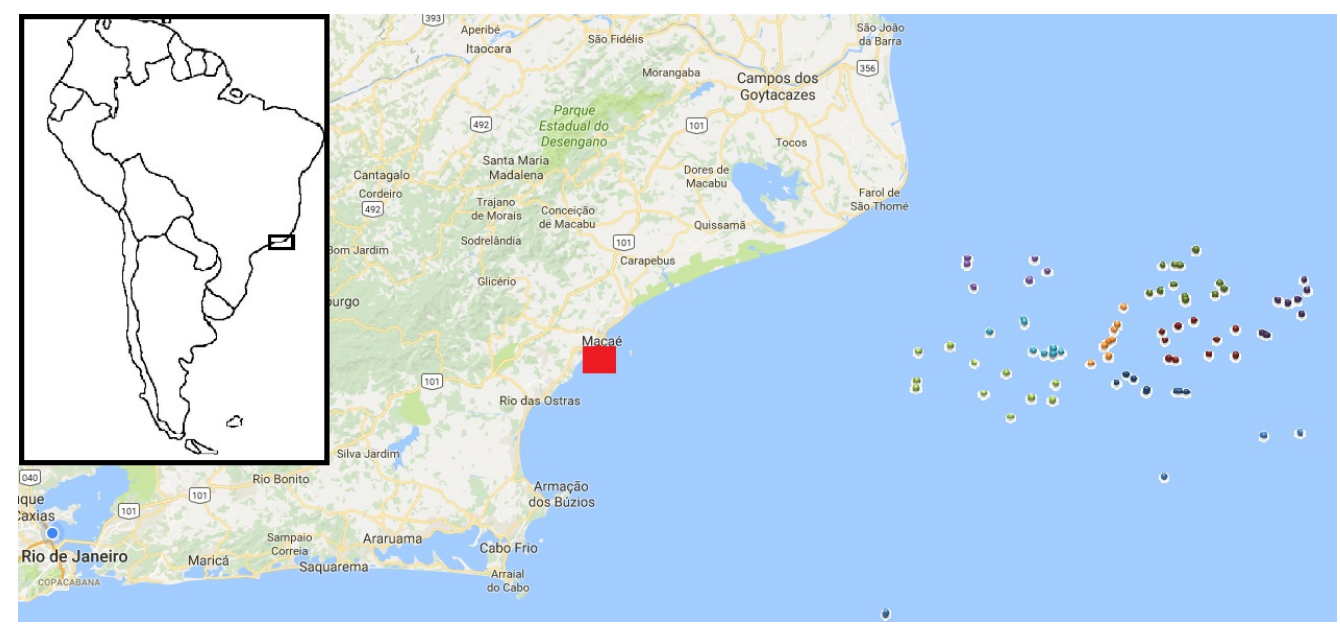

Figure 4: Supply base and offshore installations.

are assigned to routes that depart from and return to a given onshore supply base. The operational focus is the delivery of general cargo. The pickup cargo, also known as backload, is usually less than the delivery cargo and it 
is assumed that the return cargo can be placed on the deck without violating its capacity. vii) The demand is expressed by the area occupied by each cargo as found in Halvorsen-Weare and Fagerholt (2017), Halvorsen-Weare et al. (2012) and Fagerholt and Lindstad (2000). Usually, a free area is left on the deck in order to allow for any cargo handling that may be necessary. Detailed information on this subject can be found in Seixas et al. (2016). viii) No opening hours are considered for either the onshore base or the offshore unit. Offshore units are open 24 hours a day, except when performing operations that restrict cargo transfer (e.g., diving or helicopter operations) and special cases may be treated by reversing the route-visiting sequence or by changing the sequence of a few visits, resulting in a minor impact on the overall performance. Moreover, both the onshore base and the offshore units operate seven days a week, without any interruption. ix) The maximum number of offshore units that can be visited in the same route is limited by each vessel's capacity, and must not exceed eight units, as longer voyages are more sensitive to delays and to adverse weather conditions. Assigning up to eight units to a route causes the routes' duration to vary by between one day and four days approximately. Therefore, all routes' duration are less than one evaluation period or a week. $\mathrm{x}$ ) Vessels are not linked to routes and thus may not repeat the past voyages on subsequent weeks. Rather, vessels are considered as a common resource to be used whenever requested, and this is expected to produce good overall fleet allocation. xi) The port time at the supply base is calculated by considering a fixed setup time (vessel berthing time) added to the loading time, which is given by the amount of cargo to be loaded divided by an average loading rate. Given that routes cover different numbers of offshore units, the loading time is not considered fixed but, rather, dependent on the total amount of cargo to be transported. xii) Based on the actual loading rates and on the historical average amount of cargo transported, the maximum number of departures from each berth on each day is limited to two. Each departure from each berth position is nominated as a departing position. xiii) The vessel may begin loading at any time during the day. Consequently, there is a risk of not attending the imposed departing day if the loading process begins late in a day. However, in order to give flexibility to the berth planning process, a tolerance of up to 12 hours is considered. For example, the departure day of a given vessel may be considered as belonging to day $l$ even if the vessel leaves the port by 12 pm of the following day $l+1$. xiv) All parameters are known in advance. No stochastic data are used, and the weather impact on the voyage duration is 
not considered.

\section{Literature Review}

The planning of supply operations has been addressed by several authors. In Aas et al. (2009) a general overview of the supply vessel role in the oil industry is presented. Aas et al. (2007) studied the pickup and delivery problem in the offshore oil industry involving one single vessel, and considered limited storage capacity at the platforms and on the vessel. A mathematical model was proposed taking into consideration that any platform may be visited twice in order to have its demand fulfilled, given the limited storage capacity. Gribkovskaia et al. (2008) tackled the same problem, and proposed a tabu search procedure to solve larger instances. The route-planning problem with the purpose of assessing the cost impact of not being able to serve some offshore units during the night is addressed by Fagerholt and Lindstad (2000). Candidate routes were generated by considering the night closures and a set partition model was proposed including the fixed cost of using a vessel in the planning horizon.

A more complete version of the same problem is considered in HalvorsenWeare et al. (2012) by incorporating the periodicity in their model. Different from the previous contribution, in this paper the authors included the departing day in the route selection decision variable, which allowed the interval between consecutive voyages to be controlled, in accordance with a list of candidate departure patterns. A more sophisticated set partition model was proposed, based on a voyage generator, and instances with up to 14 offshore units were reported. This problem was also solved by a large neighbourhood search in Shyshou et al. (2012), and reports on solving larger instances were given. The same problem was later studied by Halvorsen-Weare and Fagerholt (2017) who proposed a new formulation based on arc-flow variables. Kisialiou et al. (2018) extended the work of Halvorsen-Weare et al. (2012) by allowing flexible departure times from the onshore base, instead of considering a fixed departure time for all voyages. The authors also dealt with the 'end of week effect', by which a vessel that started a trip by the end of a week may not be able to repeat the routes performed in the beginning of the previous week. The authors proposed an ALNS heuristic and the results were compared to a voyage-based model solved by CPLEX.

A single vessel pickup and delivery problem in the offshore industry is addressed by Cuesta et al. (2017). The problem considers that it is not 
mandatory to attend to the whole demand; however, in such cases, a cost penalty is introduced to consider the losses due to unattended demand. A mathematical model was proposed to select the sets of cargo to be transported, followed by the vessel routing. Another situation was considered, where transportation of all cargo was compulsory, even if additional vessels were incorporated into the fleet. The problem was solved by an adaptive large neighborhood search.

In the oil industry, besides the aforementioned references such as HalvorsenWeare et al. (2012) and Halvorsen-Weare and Fagerholt (2017), other contributions related to fleet sizing include Shyshou et al. (2010) who proposed a discrete event simulation model in an anchor-handling operational context. Maisiuk and Gribkovskaia (2014) studied a platform supply vessels' fleet-sizing problem under uncertainty by combining optimization and discrete event simulation. Eskandari et al. (2016) proposed a multi-objective discrete event simulation model for a supply vessel fleet-sizing problem, and Stålhane et al. (2016) developed a two-stage stochastic optimization model in a fleet-sizing problem related to maintenance activities.

The period routing problem is an important variation of the classical vehicle routing problem as many practical applications impose multiple visits to the customers during the planning period, as in Christofides and Beasley (1984) and Baptista et al. (2002). A general overview of existing models, solution approaches and applications is given in Francis et al. (2008). The integration of periodic routing with fleet sizing is also found in another class of problems known as the periodic location routing problems, which extends the period routing and fleet-sizing problem by considering location decisions. Prodon (2011), Hemmelmayr (2015) and Koç (2016) offer a general overview of existing models and solution approaches.

Berth allocation problems have been mostly investigated in the container industry, under different configurations. For example, Lim (1998) considered the problem with the continuous quayside, while Imai et al. (1997) studied the discrete quayside. Imai et al. (2001) considered that the ships arrive dynamically and in Cordeau et al. (2005) time windows for berthing the ships are imposed. In Agra and Oliveira (2018) a more complex version of the problem was considered by integrating berth planning with the planning of cranes. For a comprehensive overview, one can refer to Bierwirth and Meisel (2015). Applications in other types of terminals can be found, for example, in Ribeiro et al. (2016) and in Pratap et al. (2017). 


\section{Mathematical Model}

This section presents a mathematical model for the fleet composition and periodic routing problem with berth allocation decisions. As the model uses route selection variables based on a set of externally generated routes, a general overview of the route generation process is first given.

\subsection{Route Generation Algorithm}

The route generation process consists of enumerating all combinations of up to eight offshore units and discarding the infeasible combinations with respect to vessel capacity. In our problem, however, the units belong to predefined clusters, and no route visits offshore units belonging to different clusters. The route total demand is calculated by summing the weekly total demand of each unit present in the route divided by the week frequency of the visit to the corresponding offshore units. For each vessel class, the route is considered feasible if the calculated total demand is less than or equal to the vessel capacity. As the offshore units operate uninterrupted during the night, one may solve the inherent TSP problem to determine the optimal route sequence, and the corresponding cost and duration. Figure 5 gives a general overview of the generation process.

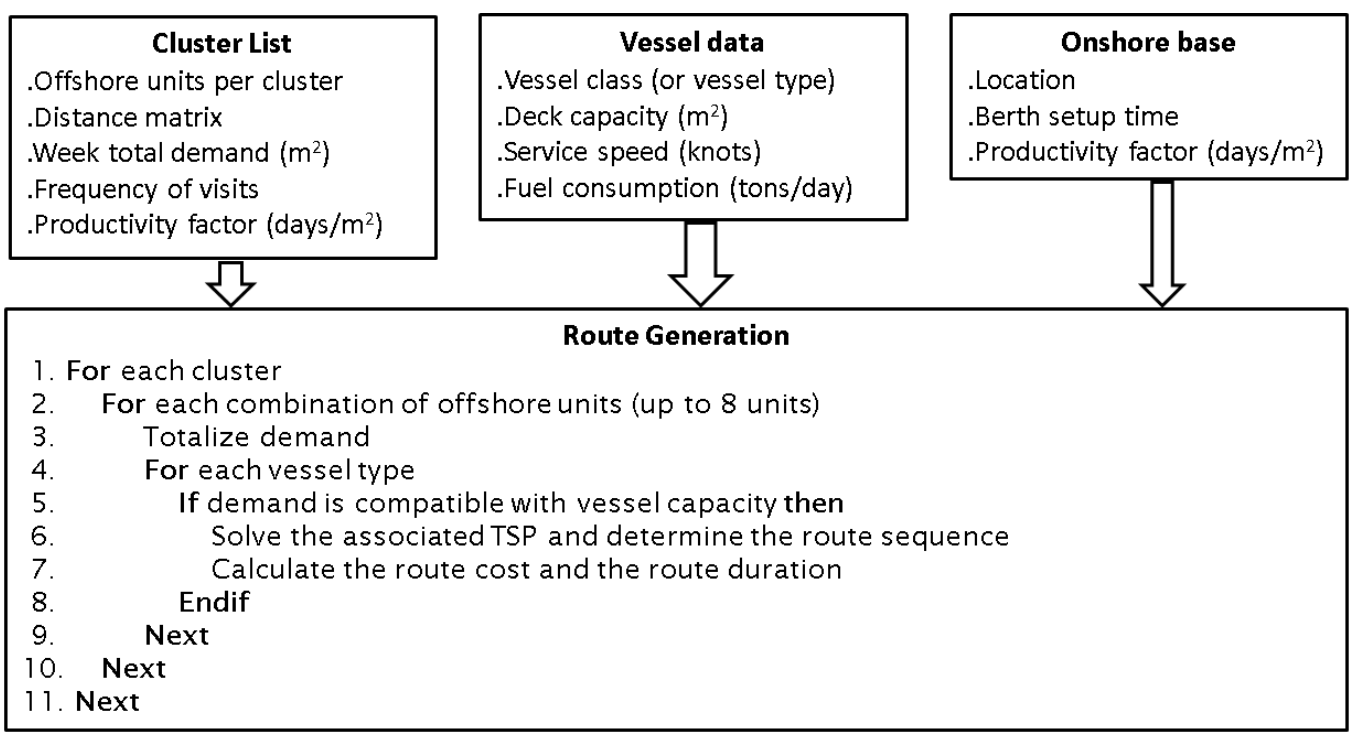

Figure 5: Route generation procedure. 
As a periodic routing problem is being solved, if the routes depart in an evenly spread configuration from the supply base then it is not expected that the offshore units are serviced by two different routes at the same time. In case this condition occurs, it is always possible to eliminate the collision by reversing the visit sequence of one of the routes.

\subsection{Sets, Indices, Parameters and Decision Variables}

The following sets and indices are used in the mathematical model: $V$ - set of vessel classes (index $v$ ); $J$ - set of offshore units (index $j$ ); $R$ - set of routes (index $r$ ); $R_{v}$ - subset of routes that vessels of class $v$ can sail $\left(R_{v} \subset R\right) ; S$ set of departure patterns (i.e. departure days) from the supply base (index $s) ; S_{j}$ - subset of departure patterns compatible with the frequency of visits imposed by offshore unit $j\left(S_{j} \subset S\right)$; $T$ - planning horizon of the vessels (indices $l$ and $t, t: 1 . .14) ; L$ - planning horizon of the offshore units $(L \subset T$, index $l, l: 1 . .7) ; B$ - set of berths (index $b) ; P$ - set of departures per day for each berth (index $p$ ). For the sake of simplicity, as each vessel is always assigned to a pair (berth, departing position) at the onshore base, a berth position $(b, p)$ notation is used.

Parameters: $F V_{v}$ - fixed cost of vessels belonging to class $v ; C R_{v r}$ - route $r$ cost of vessels belonging to class $v ; D_{j}$ - offshore unit $j$ demand $\left(m^{2}\right)$ given by the mean plus two times the standard deviation of the weekly demand divided by the imposed frequency of visits (in order to cover possible demand variations); $A_{r j}^{0}$ - binary parameter that is 1 if route $r$ visits offshore unit $j$, and 0 otherwise; $A_{s l}^{1}$ - binary parameter that is 1 if the departure pattern $s$ has a departure on day $l$, and 0 otherwise; $S T_{r v}$ - route $r$ sailing time of vessels belonging to class $v$; $P B$ - productivity factor (days $/ m^{2}$ ) for cargo handling at the onshore base; $P O_{j}$ - productivity factor (days $/ \mathrm{m}^{2}$ ) for cargo handling at offshore unit $j ; S B$ - berth set up time (average time for approaching and mooring); $L L_{l b}$ - lower time limit for starting loading of a vessel on berth $b$ and day $l$; and $L U_{l b}$ - upper time limit for finishing the loading of a vessel on berth $b$ and day $l$. These parameters $\left(L L_{l b}\right.$ and $\left.L U_{l b}\right)$ allow for the modeling of the tolerance in the departure time of a vessel being loaded at berth position $(b, p)$. For example, $L L_{l b}$ for day 5 is equal to 4.0 and $L U_{l b}$ is set to 5.5, thus allowing a vessel to depart up to $12 \mathrm{pm}$ of day 6 .

Decision variables: $n_{v}$ - number of utilized vessels of class $v ; b_{s j}$ - binary variable that is 1 if departure pattern $s$ is chosen for offshore unit $j$, and 0 otherwise (refer to Figure 3 for the list of departure patterns); $x_{v b p r l}$ - binary variable that is 1 if a vessel of class $v$ departs from the berth position $(b, p)$ 
on day $l$ to execute route $r$, and 0 otherwise; $c_{v b p t_{1} t_{2}}$ - binary variable that is 1 if a vessel of class $v$ departing from the berth position $(b, p)$ on day $t_{1}$ is still operating its route by day $t_{2}$, and 0 otherwise; $s l_{b p l}$ - loading time spent on berth position $(b, p)$ on day $l ; s_{b p l}$ - instant of time that the loading operation of the berth position $(b, p)$ on day $l$ is concluded and the vessel starts the voyage; and $t_{b p l}$ - round trip voyage duration of the vessel that occupies the berth position $(b, p)$ on day $l$.

\subsection{Mathematical Model}

The mathematical model is defined as:

$$
\min Z=\sum_{v \in V} F V_{v} n_{v}+\sum_{v \in V} \sum_{b \in B} \sum_{p \in P} \sum_{r \in R_{v}} \sum_{l \in L} C R_{v r} x_{v b p r l}
$$

subject to:

$$
\begin{aligned}
& \sum_{s \in S_{j}} b_{s j}=1 \quad j \in J \\
& \sum_{v \in V} \sum_{b \in B} \sum_{p \in P} \sum_{r \in R_{v}} x_{v b p r l} A_{r j}^{0} \geq \sum_{s \in S_{j}} b_{s j} A_{s l}^{1} \quad l \in L, j \in J \\
& \sum_{v \in V} \sum_{r \in R_{v}} x_{v b p r l} \leq 1 \quad l \in L, b \in B, p \in P \\
& \sum_{v \in V} \sum_{r \in R_{v}} x_{v b 2 r l} \leq \sum_{v \in V} \sum_{r \in R_{v}} x_{v b 1 r l} \quad l \in L, b \in B \\
& s_{b 11} \geq s l_{b 11} \quad b \in B \\
& s_{b 2 l} \geq s_{b 1 l}+s l_{b 2 l} \quad b \in B, l \in L \\
& s_{b 1(l+1)} \geq s_{b 2 l}+s l_{b 1(l+1)} \quad b \in B, l \in L, l \geq 2 \\
& L L_{l b}+s l_{b p l} \leq s_{b p l} \leq L U_{l b} \quad l \in L, b \in B, p \in P \\
& s l_{b p l} \geq \sum_{v \in V} x_{v b p r l}\left(S B+\sum_{j \in J: A_{r j}^{0}=1} D_{j} P B\right) \\
& l \in L, r \in R, b \in B, p \in P
\end{aligned}
$$




$$
\begin{aligned}
& t_{b p l} \geq \sum_{v \in V} x_{v b p r l}\left(S T_{r v}+\sum_{j \in J: A_{r j}^{0}=1} D_{j} P O_{j}\right) \\
& l \in L, r \in R, b \in B, p \in P \\
& \sum_{t_{2}=l}^{|T|} c_{v b p l t_{2}} \geq s_{b p l}+t_{b p l}-l-\left(1-c_{v b p l l}\right)|T| \\
& v \in V, b \in B, p \in P, l \in L \\
& c_{v b p l l} \geq x_{v b p r l} \\
& l \in L, v \in V, r \in R, b \in B, p \in P \\
& c_{v b p t_{1} t_{2}}=0 \\
& v \in V, b \in B, p \in P, t_{1} \in L, t_{2} \in L, t_{2}<t_{1} \\
& c_{v b p t_{1}\left(t_{2}+1\right)}-c_{v b p t_{1} t_{2}} \leq 0 \\
& v \in V, b \in B, p \in P, t_{1} \in L, t_{2} \in T, t_{2} \geq t_{1} \\
& c_{v b p\left(t_{1}+|L|\right)\left(t_{2}+|L|\right)}=c_{v b p t_{1} t_{2}} \\
& v \in V, b \in B, p \in P, t_{1} \in L, t_{2} \in L \\
& n_{v} \geq \sum_{t_{1} \in T} \sum_{b \in B} \sum_{p \in P} c_{v b p t_{1} t_{2}} \quad v \in V, t_{2} \in T \\
& n_{v} \in \mathbb{Z}^{+}, b_{s j} \in\{0,1\}, x_{v b p r l} \in\{0,1\}, c_{v b p t_{1} t_{2}} \in\{0,1\}, \\
& s_{b p l} \in \mathbb{R}^{+}, s l_{b p l} \in \mathbb{R}^{+}, t_{b p l} \in \mathbb{R}^{+} .
\end{aligned}
$$

The objective function (1) minimizes the vessels' fixed costs and the routing costs. Constraint (2) ensures that one departure pattern is assigned to each offshore unit. Constraint (3) ensures that there is at least one vessel departing on each day belonging to the selected departure days of each offshore unit. Constraint (4) limits to at most one the number of routes departing per day for each berth position. Constraint (5) forces that berth position two is only assigned if position one was used. Constraint (6) initializes the berth's departure time based on the berth's loading time of the first day. Constraint (7) ensures that the departure time for position two of any berth depends on the position one departure time added to the position two loading time. Constraint (8) ensures that the departure time for berth position 1 is greater than the instant in which the loading operation of the previous 
day was concluded, for $l \geq 2$. Constraint (9) indicates that the departure time for any berth position is within the time window defined by $L L_{l b}$ and $L U_{l b}$. Constraint (10) ensures that the operating time of any berth position must be greater than the sum of the vessel's set up time and the loading time. Constraint (11) ensures that the vessel's round trip duration must be greater than the sum of the vessel's sailing time and the cargo handling time at each visited offshore unit. Constraint (12) ensures that a binary counter is activated (i.e. equal to 1), for the time period that a vessel is in use. Constraint (13) associates the binary counter with the $x_{v b p r l}$ variable. Constraint (14) ensures that the binary counter is not activated in any period $t_{2}$ prior to a candidate's departing day $t_{1}$. Constraint (15) links the counter variable of one day to its previous day. Constraint (16) replicates a vessel class assignment for the subsequent period. Constraint (17) calculates the number of vessels per class as the maximum number of vessels in use at the same time. Constraint (18) define the variables' domain. Constraints (12) to (17) ensure that voyages of the same vessel should not overlap in time.

An example of the calculation of variable $c_{v b p t_{1} t_{2}}$ in terms of the departure day $t_{1}$ (rows) and the respective days that the vessel is in use $t_{2}$ (columns) is given in Table 1. The example is based in a case where a vessel of class 1 is assigned to perform a route that departs on day 5 from berth 1 and position 1. Besides, it is assumed that the vessel should be in use for 3.6 days, according to (12). Therefore, the minimum number of vessels for each class $n_{v}$ must be equal to the maximum number of vessels for the respective class in use at any time $t_{2}$ (the columns in Table 1), as indicated in (17).

In order to illustrate the use of parameters $L L_{l b}$ and $L U_{l b}$ consider, for instance, a loading operation that takes place on day 5 , where $L L_{l b}=4.0$ and $L U_{l b}=5.5$. If two vessels are assigned to berth 2 with loading times of 0.5 and 0.6 (for positions 1 and 2 respectively), and assuming that there is no loading on berth 2 on the previous day then, according to (10): $s l_{215} \geq 0.5$ and $s l_{225} \geq 0.6$. Constraint (9) defines the interval for departure of each position as $4.0+0.5 \leq s_{215} \leq 5.5$ and $4.0+0.6 \leq s_{225} \leq 5.5$. Constraint (7) ensures that position 2 only starts after position $1, s_{225} \geq s_{215}+s l_{215}$ and, if we use the minimum values, the constrain is $s_{225} \geq 4.5+0.6$. This means that any vessel assigned for berth position 2 starts loading on day 4.5 (or after) and departs from the harbor on day 5.1 (or after). According to constraint (8) the departure for the next day (day 6) for berth 2 should consider that the loading in position 1 starts after vessel from position 2 on the previous day has departed: $s_{216} \geq 5.1+s l_{216}$. By the use of the upper limit extended to 
Table 1: Example of the calculation of variable $c_{v b p t_{1} t_{2}}$.

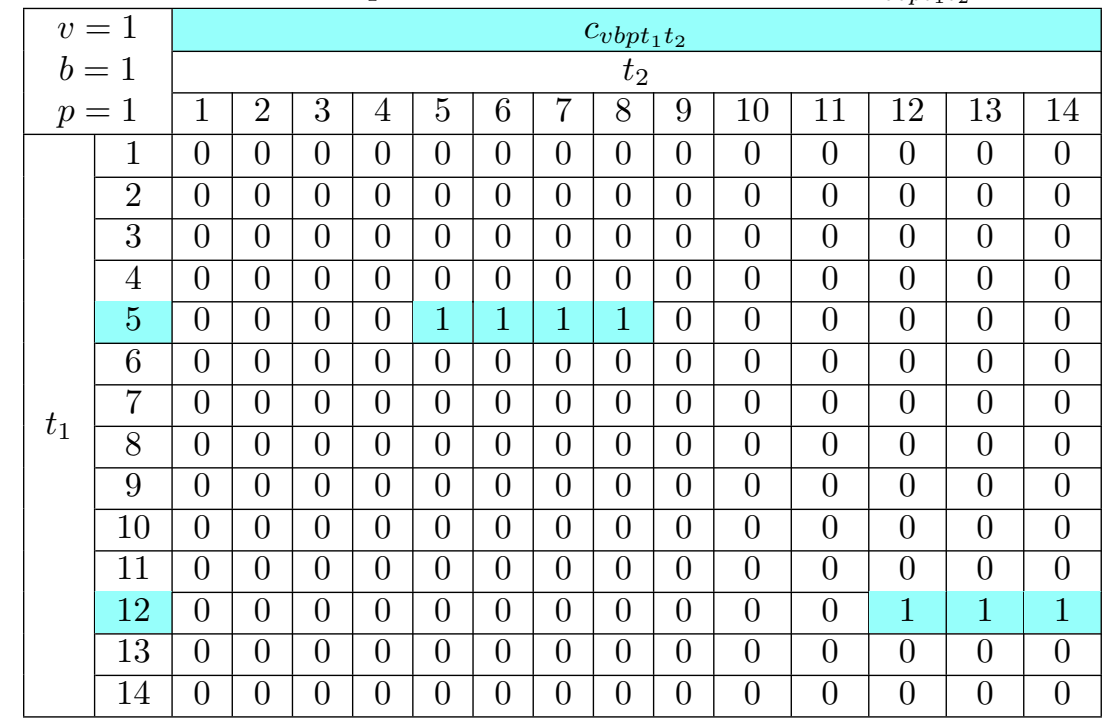

the next day it is possible to accommodate better berth allocation, without compromising the evenly spread departures from the harbor. This is a very important relaxation in the case of a busy harbor.

\section{Solution Strategy}

The model proposed for the fleet-sizing and periodic routing problem with berth allocation decisions is difficult to solve, due to its combinatorial nature. In order to achieve a good-quality solution or even the optimal solution, the problem is solved in four steps. The initial steps simplify many important constraints, which are progressively incorporated. The idea is that the solution of any given step provides a lower bound to the following step and, in the case of the fourth step, a bound is provided for the complete model. Although the complexity increases with each step, the informed bounds are meant to reduce the processing time. In the following, each step is detailed.

Step 1 - The berth allocation constraints are relaxed and a limit on the maximum number of departures (i.e. routes) per day is imposed as two times the number of berths, as no more than two departures per berth are expected to occur in each day. The routing costs are also eliminated from the objective function. Lastly, instead of calculating the number of vessels as proposed by constraint (17), the vessels' operating time (in days) are summed, for each 
class, and divided by seven (one week). This is an approximation that is refined in Step 2, taking into account the vessels' temporal distribution.

Step 2 - The difference from Step 1 is the refinement in the fleet-sizing process. The fleet is now defined by the maximum number of vessels in use in each day, for each class, as in constraint (17). The fleet cost of Step 2 cannot be inferior to the Step 1 fleet cost, and this is represented by a constraint.

Step 3 - The routes selected in Step 2 are not necessarily optimal if the routing costs are considered, despite being able to yield the lowest fleet cost, which was accurately calculated in Step 2. In Step 3, the same model proposed for Step 2 is considered except that the routing costs are incorporated in the objective function. As in the previous case, the fleet cost of Step 3 cannot be inferior to the fleet cost of Step 2, and a constraint is added to the model in this respect.

Step 4 - The problem solved in Step 3 is complete except for the berth allocation decisions, which are considered in this step. The routes from the Step 3 solution are used as input to Step 4.

\subsection{Step 1 Mathematical Model}

In order to solve Step 1, new decision variables are introduced to allow working with the simplified model, $w_{v r l}$ - binary variable that is 1 if a vessel of class $v$ executes route $r$ on day $l$, and 0 otherwise; and $t c_{v r l}$ - integer variable that registers the cycle time for a vessel of class $v$ when executing route $r$ on day $l$. The cycle time is the time span between the beginning of the vessel loading at the harbour until its return to the onshore base, after

performing a route. $Z_{1}$ is the value of the objective function, and $Z_{1}$ is the lower-bound value obtained with the model processing. The model can be stated as follows:

$$
\min Z_{1}=\sum_{v \in V} F V_{v} n_{v}
$$

subject to:

$$
\begin{gathered}
\sum_{s \in S_{j}} b_{s j}=1 \quad j \in J \\
\sum_{v \in V} \sum_{r \in R_{v}} w_{v r l} A_{r j}^{0} \geq \sum_{s \in S_{j}} b_{s j} A_{s l}^{1} \quad l \in L, j \in J \\
\sum_{v \in V} \sum_{r \in R_{v}} w_{v r l} \leq|B||P| \quad l \in L
\end{gathered}
$$




$$
\begin{gathered}
t c_{v r l} \geq w_{v r l}\left[S B+S T_{r v}+\sum_{j \in J: A_{r j}^{0}=1}\left(P B+P O_{j}\right) D_{j}\right] \\
l \in L, v \in V, r \in R_{v} \\
n_{v} \geq \sum_{l \in L} \sum_{r \in R_{v}} t c_{v r l} /|L| \quad v \in V \\
n_{v} \in \mathbb{Z}^{+}, \quad t c_{v r l} \in \mathbb{Z}^{+}, \quad b_{s j} \in\{0,1\}, \quad w_{v r l} \in\{0,1\} .
\end{gathered}
$$

The objective function (19) minimizes vessels' fixed costs based on their average use during the planning period. Constraint (20) ensures that one departure pattern is assigned for each offshore unit. Constraint (21) ensures that there is at least one vessel departing on each day belonging to the selected departure pattern of each offshore unit. Constraint (22) limits the number of departures per day based on the number of available berth positions. Constraint (23) determines the cycle time for a vessel of class $v$ when assigned to route $r$ on day $l$. Constraint (24) calculates the number of vessels per class, based on the average utilization in the considered period (one week). Constraint (25) defines the variables' domain.

\subsection{Step 2 Mathematical Model}

In order to solve Step 2, the following variable is needed: $y_{v_{r} t_{1} t_{2}}$ - binary variable that is 1 if a vessel of class $v$ departing on day $t_{1}$ is still operating on route $r$ by day $t_{2}$, and 0 otherwise. This variable plays a similar role as $c_{v b p t_{1} t_{2}}$ in the complete model, and allows for computing the maximum number of class $v$ vessels in use. $Z_{2}$ is the value of the objective function, and $Z_{2}$ is the lower-bound value obtained with the model processing. The model can be stated as:

$$
\min Z_{2}=\sum_{v \in V} F V_{v} n_{v}
$$

subject to:

$$
\begin{gathered}
\sum_{s \in S_{j}} b_{s j}=1 \quad j \in J \\
\sum_{v \in V} \sum_{r \in R_{v}} w_{v r l} A_{r j}^{0} \geq \sum_{s \in S_{j}} b_{s j} A_{s l}^{1} \quad l \in L, \quad j \in J
\end{gathered}
$$




$$
\begin{aligned}
& \sum_{v \in V} \sum_{r \in R_{v}} w_{v r l} \leq|B||P| \quad l \in L \\
& \sum_{t_{2}=t_{1}}^{|T|} y_{v r t_{1} t_{2}} \geq w_{v r l}\left[S B+S T_{r v}+\sum_{j \in J: A_{r j}^{0}=1}\left(P B+P O_{j}\right) D_{j}\right] \\
& l \in L, t_{1} \in L, t_{2} \in T, v \in V, r \in R \\
& y_{v r l l} \geq w_{v r l} \quad l \in L, v \in V, r \in R_{v} \\
& y_{v_{v r} t_{2}}=0 \quad t_{1} \in L, t_{2} \in L, v \in V, r \in R_{v}, t_{2}<t_{1} \\
& y_{v r t_{1}\left(t_{2}+1\right)}-y_{\text {vrt }_{1} t_{2}} \leq 0 \quad t_{1} \in L, t_{2} \in T, v \in V, r \in R, t_{2} \geq t_{1} \\
& y_{v r\left(t_{1}+|L|\right)\left(t_{2}+|L|\right)}=y_{v r t_{1} t_{2}} \quad t_{1} \in L, t_{2} \in L, v \in V, r \in R \\
& n_{v} \geq \sum_{t_{1} \in T} \sum_{r \in R} y_{v r t_{1} t_{2}} \quad v \in V, t_{2} \in T \\
& \sum_{v \in V} F V_{v} n_{v} \geq \underline{Z_{1}} \\
& n_{v} \in \mathbb{Z}^{+}, b_{s j} \in\{0,1\}, w_{\text {vrl }} \in\{0,1\}, y_{\text {vrt }_{1} t_{2}} \in\{0,1\} .
\end{aligned}
$$

The objective function (26) minimizes the vessels' fixed costs based on the maximum number of vessels that is required for each vessel class in the planning period. Constraints (27) to (29) have the same purposes as stated in the previous model. Constraint (30) ensures that a binary counter is activated (i.e. equal to 1 ), for the time period that a vessel of class $v$ is executing route $r$. Constraint (31) forces variable $y_{v_{r} t_{1} t_{2}}$ to be 1 if a vessel of class $v$ departs from the port on day $t_{1}$ to execute route $r$. Constraint (32) ensures that the binary counter is not activated in any period $t_{2}$ prior to a candidate departing day $t_{1}$. Constraint (33) links the counter variable of one day to its previous day. Constraint (34) replicates a vessel class assignment for the subsequent period. Constraint (35) calculates the number of vessels per 
class, as the maximum number of vessels in use at the same time. Constraint (36) imposes a lower-bound value on the fleet cost, based on the Step 1 lower bound. Constraint (37) defines the variables' domain.

\subsection{Mathematical Model for Step 3}

In Step 3, the same model from Step 2 is solved except that the objective function incorporates the routing costs. The solution found in Step 2 is used as an initial solution, and also provides a lower bound to the objective function. $Z_{3}$ is the value of the objective function, and $Z_{3}$ is the lower-bound value obtained with the model processing. The model is stated as:

$$
\min Z_{3}=\sum_{v \in V} F V_{v} n_{v}+\sum_{v \in V} \sum_{r \in R_{v}} \sum_{l \in L} C R_{v r} w_{v r l}
$$

subject to:

(27) to $(35),(37)$

$$
\sum_{v \in V} F V_{v} n_{v} \geq \underline{Z_{2}}
$$

The objective function (38) minimizes the vessels' fixed costs and the routing costs, and constraint (39) is the lower bound.

\subsection{Step 4 Mathematical Model}

In Step 4, the berth allocation decisions are made based on input from the set of routes that were selected in Step 3. These routes are represented by the subset $R_{3} \subset R$. In Step 4, the complete model is complemented by the indicated route selection constraints and the lower-bound constraints. $Z_{4}$ is the value of the objective function, and $Z_{4}$ is the lower-bound value obtained with the model processing. The model can be stated as:

$$
\min Z_{4}=\sum_{v \in V} F V_{v} n_{v}+\sum_{v \in V} \sum_{b \in B} \sum_{p \in P} \sum_{r \in R_{v}} \sum_{l \in L} C R_{v r} x_{v b p r l}
$$

subject to:

(2) to (18)

$$
\sum_{v \in V} \sum_{b \in B} \sum_{p \in P} \sum_{l \in L} x_{v b p r l}=1 \quad r \in R_{3}
$$




$$
\begin{gathered}
\sum_{v \in V} \sum_{b \in B} \sum_{p \in P} \sum_{l \in L} x_{v b p r l}=0 \quad r \in R \backslash R_{3} \\
\sum_{v \in V} F V_{v} n_{v} \geq \underline{Z_{2}} \\
\sum_{v \in V} F V_{v} n_{v}+\sum_{v \in V} \sum_{b \in B} \sum_{p \in P} \sum_{r \in R_{v}} \sum_{l \in L} C R_{v r} x_{v b p r l} \geq \underline{Z_{3}}
\end{gathered}
$$

The objective function (40) minimizes the fleet's fixed costs and the routing costs. Constraints (41) and (42) assign the routes obtained in Step 3 and discard all the others. Constraint (43) defines that the fleet cost must be greater than or equal to the Step 2 lower bound, and constraint (44) defines that the objective function must be greater than or equal to the Step 3 lower bound.

In the problem description, it was considered that a vessel meant to depart on day $l$ could be delayed up to 12 hours, thus leaving the port any time before $12 \mathrm{pm}$ of day $l+1$. This could happen in situations where a vessel was not able to start loading earlier on day $l$, due to limited berth capacity. The berth scheduling process therefore focuses on how to accommodate all the vessels in such a way that all departures fit in a one-week period. The berth scheduling may have a direct impact on the fleet size as well. The $c_{v b p t_{1} t_{2}}$ binary decision variable is equal to 1 each day that a vessel of class $v$ is in use, after leaving the port. However, according to constraint (12), the sum of the $c_{v b p t_{1} t_{2}}$ variables is influenced by the departure time, which is given by $s_{b p l}$. For example, if the route duration is 2.3 days and the departure time is 0.5 , then the assigned vessel is in use until instant 2.8 , and three $c_{v b p t_{1} t_{2}}$ variables are set to 1 ; but, if the departure takes place in instant 0.9 , the assigned vessel is in use until instant 3.2, and therefore four $c_{v b p t_{1} t_{2}}$ variables are set to 1 . In this case, the maximum number of vessels in use, which is assessed by constraint (17), may indicate a different fleet.

If the solution found in Step 3 indicates a number of departures per day that are inferior or equal to the number of available berths, then the Step 4 solution is the same as for Step 3. However, as this may not be the case, in Step 4, the complete model is processed with the following simplifications in order to make the model processing more tractable: the routes that were generated in Step 3 are retained, and the decisions regarding the departure pattern (i.e. the departing days for each offshore unit) and the vessel class 
that is assigned to each route are released. This means that the routes selected in Step 3 can be sailed by a vessel from a different class and on a different day other than the ones established in that previous step, thus giving more flexibility to the solution procedure. Although this procedure may not lead to the optimal solution, good-quality solutions are expected to be obtained which would not be otherwise possible if one was to resort to solving the complete model. Finally, the objective function of Step 3 is a natural lower bound on the objective function of Step 4, and the Step 4 solution provides an upper bound to the complete model.

\section{Case Study}

The model applicability is demonstrated by real-based cases obtained from a Brazilian oil company, from its operations at Campos Basin (Figure 4). Four cases are presented: these are cases C10, C15, C41 and C79, having 10, 15, 41 and 79 offshore units, respectively. In cases $\mathrm{C} 10$ and $\mathrm{C} 15$, routes are generated considering all possible combinations for all units. In cases C41 and C79, routes are generated considering all possible combinations of the units belonging to their clusters (i.e. groups of offshore units).

In the offshore E\&P one finds permanent units such as the production platforms that remain fixed in their positions for as long as 25 years, and mobile units, related to drilling rigs and maintenance platforms, that are constantly moved from one oilfield to another. Those mobile units usually require a different service level in terms of frequency of visits and different types of cargo when compared to the permanent units. Therefore, an oil company may consider grouping near-by permanent units and near-by mobile units in order to form clusters. Another policy is to group near-by units irrespective of their type (permanent or mobile). Instances were generated to compare these two policies. Those with the suffix $\mathrm{S}$ indicate that the mobile units are segregated from the permanent units.

Table 2 presents some key features regarding each instance. The columns indicate the following data: OU - number of offshore units to be served; Routes - number of generated routes to be used as input data in the mathematical model; Clusters - number of clusters (groups of platforms to be serviced together and apart from the others) for each instance; OUmax maximum number of offshore units served in a cluster; OUmin - minimum number of offshore units served in a cluster; Rmax - maximum number of 
generated routes in a cluster; and Rmin - minimum number of generated routes considered in a cluster.

Table 2: Instances

\begin{tabular}{|l|r|r|r|r|r|r|r|}
\hline Case & OU & Routes & Clusters & OUmax & OUmin & Rmax & Rmin \\
\hline C10 & 10 & 912 & 1 & 10 & 10 & 912 & 912 \\
\hline C15 & 15 & 10,021 & 1 & 15 & 15 & 10,021 & 10,021 \\
\hline C41 & 41 & 1,372 & 5 & 11 & 6 & 822 & 47 \\
\hline C41S & 41 & 438 & 7 & 8 & 4 & 199 & 14 \\
\hline C79 & 79 & 2,168 & 9 & 12 & 6 & 882 & 47 \\
\hline C79S & 79 & 1,107 & 12 & 10 & 4 & 315 & 14 \\
\hline
\end{tabular}

Case C10 is the easiest to solve and is used to verify the effectiveness of the step solution strategy performance, when compared to the use of the complete model. Case C15 allows us to demonstrate the difficulty of solving an instance with a high overall number of routes. Cases C41 and C79 are intermediate and hard-to-solve instances, respectively, and allow for assessing the proposed solution strategy. Not only the overall number of routes defines hard-to-solve instances. A large number of offshore units divided into clusters might produce hard-to-solve instances, even with a limited number of routes in each cluster. This is clearly demonstrated in the results obtained for cases C79.

For all cases, four berths were considered available with a maximum of two departures per berth each day. A tolerance of half a day was allowed for the vessel departure time, to allow better berth utilization.

All models were implemented in $\mathrm{C}++$ and solved by Gurobi. The results were obtained using a $2.27 \mathrm{GHz} \operatorname{Intel}(\mathrm{R}) \mathrm{Xeon}(\mathrm{R}) \mathrm{E} 5520$, with 16 cores, 48 GB RAM, and solved by GUROBI 7.0.2. The routes were previously generated in a Excel spreadsheet using VBA. The processing time for route generation varies from 30 to 2,100 seconds depending on the instance. The code for the route generation has not been developed for optimal performance and its processing time has been disregarded from the computational study. The maximum time limit of 99,000 seconds has been set for each run, in order to verify how far it was possible to go with the proposed solution strategy.

In the tables used to present the main results, the lines represent the following: OF - objective function value; VC - vessels' costs; RC - routing costs; V0 - number of PSV4500 vessels; V1 - number of PSV3000 vessels; V2 - number of PSV1500 vessels; NR - total number of sailed routes; ND - maximum number of departures at any day; B - number of berths; GAP 
- percentage difference from the upper and lower bounds given by Gurobi; Time - processing time in seconds; and CTime - cumulative processing time in seconds for the steps in the proposed solution strategy, and the processing time in seconds in case of the complete model. As for the columns, they are organized according to each step proposed in the solution strategy: S1 refers to Step 1, S2 refers to Step 2, and so on. In some cases, Step 4 is tested for a number of berths other than four, indicated in line B. Column CM refers to the complete model, defined in 4.3.

\subsection{Results for Cases $C 10$ and $C 15$}

The results for cases $\mathrm{C} 10$ and $\mathrm{C} 15$ are provided in Table 3. It can be noticed that case C10 presented no variation in the number of vessels, with each solution step. The maximum number of departures on the same day decreased significantly from Step 1 to Step 2 because of the vessel usage consideration. However, a considerable difference can be noticed for the processing time. The complete model took 84,340 seconds to process, while the total processing time for the solution strategy was 115 seconds.

In case C15 the fleet varied with each step, except for the last one. In Step 1 , two vessels were needed to perform five routes. However, when the fleet was calculated more accurately, three small vessels were chosen to perform 11 routes. In Step 3, as the routing costs were computed, two large vessels were selected to perform four routes. The fleet cost increased from 75 to 76 , but this was compensated for by a reduction in the routing costs. In Step 2, the routing costs, which are not shown in Table 3, were 8.04, and in Step 3, the routing costs were 4.37, yielding an overall cost reduction of 2.67. As for the complete model, an out-of-memory error interrupted the processing at instant 21,289 seconds, with a $57.90 \%$ gap. The proposed solution strategy thus proved to be efficient and an optimal solution could be found in 6,812 seconds. For both cases $\mathrm{C} 10$ and $\mathrm{C} 15$, given that optimal solutions were attained at Steps 1 to 3, and that there were enough berths to accommodate all the departures generated in Step 3, the Step 4 solutions are also optimal to the complete model.

\subsection{Results for Cases $C 41$ and $C 41 S$}

Table 4 presents the results for cases C41 and C41S, without and with segregation regarding servicing mobile units apart from the permanent units, respectively. Different from cases $\mathrm{C} 10$ and C15, these cases were also tested for two and three berths. It can be noticed that in case $\mathrm{C} 41$, the number 


Table 3: Results for cases $\mathrm{C} 10$ and C15
\begin{tabular}{|l|c|c|c|c|c|}
\hline C10 & S1 & S2 & S3 & S4 & CM \\
\hline OF & 50 & 50 & 53.77 & 53.77 & 53.77 \\
VC & 50 & 50 & 50 & 50 & 50 \\
RC & - & - & 3.77 & 3.77 & 3.77 \\
V0 & 0 & 0 & 0 & 0 & 0 \\
V1 & 0 & 0 & 0 & 0 & 0 \\
V2 & 2 & 2 & 2 & 2 & 2 \\
NR & 6 & 7 & 6 & 6 & 6 \\
ND & 3 & 1 & 1 & 2 & 1 \\
B & 4 & 4 & 4 & 4 & 4 \\
Gap & 0 & 0 & 0 & 0 & 0 \\
Time & 4 & 11 & 16 & 84 & 84,340 \\
CTime & 4 & 15 & 31 & 115 & 84,340 \\
\hline \hline C15 & S1 & S2 & S3 & S4 & CM \\
\hline OF & 71 & 75 & 80.37 & 80.37 & $89.43^{\dagger}$ \\
VC & 71 & 75 & 76 & 76 & 83 \\
RC & - & - & 4.37 & 4.37 & 6.43 \\
V0 & 1 & 0 & 2 & 2 & 0 \\
V1 & 1 & 0 & 0 & 0 & 1 \\
V2 & 0 & 3 & 0 & 0 & 2 \\
NR & 5 & 11 & 4 & 4 & 9 \\
ND & 1 & 2 & 1 & 1 & 2 \\
B & 4 & 4 & 4 & 4 & 4 \\
Gap & 0 & 0 & 0 & 0 & $57.90 \%$ \\
Time & 1,862 & 4,448 & 496 & 6 & 21,289 \\
CTime & 1,862 & 6,310 & 6,806 & 6,812 & 21,289 \\
\hline
\end{tabular}

$\dagger$ Objective function value not proven to be optimal.

of vessels remained the same, except for the case with two available berths. The maximum number of departures were four and three, for cases C41 and C41S, respectively, and Steps 1 to 3 were optimally solved. Therefore, as with cases $\mathrm{C} 10$ and $\mathrm{C} 15$, the Step 4 solutions are also optimal. In order to test if the solution would change in the case that fewer berths were available, the Step 4 model was run considering three and two berths, and the results are presented in Table 4 in columns $\mathrm{S} 4(3 \mathrm{~B})$ and $\mathrm{S} 4(2 \mathrm{~B})$, respectively.

In case $\mathrm{C} 41 \mathrm{~S}$, the results for three berths were omitted, as the maximum number of departures in Step 3, with four available berths, was three and, therefore, the solution would not change. Also, when only two berths were available, the solution remained the same. As for the complete model processed with four berths, solutions with poor lower bounds were obtained, 
Table 4: Results for cases $\mathrm{C} 41$ and $\mathrm{C} 41 \mathrm{~S}$

\begin{tabular}{|l||c|c|c|c|c|c|c|}
\hline C41 & S1 & S2 & S3 & S4 & S4(3B) & S4(2B) & CM \\
\hline OF & 243 & 243 & 256.59 & 256.59 & 256.59 & $270.20^{\dagger}$ & $289.23^{\dagger}$ \\
RC & 243 & 243 & 243 & 243 & 243 & 256 & 276 \\
V0 & - & - & 13.59 & 13.59 & 13.59 & 14.20 & 13.23 \\
V1 & 4 & 4 & 4 & 4 & 4 & 5 & 4 \\
V2 & 2 & 2 & 2 & 2 & 2 & 2 & 3 \\
NR & 1 & 1 & 1 & 1 & 1 & 0 & 1 \\
ND & 19 & 19 & 19 & 19 & 19 & 19 & 18 \\
B & 4 & 4 & 4 & 4 & 4 & 3 & 4 \\
Gap & 4 & 4 & 4 & 4 & 3 & 2 & 4 \\
Time & 0 & 0 & 0 & 0 & 0 & $3.1 \%$ & $62.6 \%$ \\
CTime & 21 & 41 & 77 & 348 & 173 & 99,000 & 99,000 \\
\hline C41S & S1 & 62 & 139 & 487 & 312 & 99,139 & 99,000 \\
\hline OF & 248 & 248 & 261.99 & 261.99 & - & 261.99 & $305.00^{\dagger}$ \\
VC & 248 & 248 & 248 & 248 & - & 248 & 291 \\
RC & - & - & 13.99 & 13.99 & - & 13.99 & 14.00 \\
V0 & 5 & 5 & 5 & 5 & - & 5 & 7 \\
V1 & 1 & 1 & 1 & 1 & - & 1 & 0 \\
V2 & 1 & 1 & 1 & 1 & - & 1 & 1 \\
NR & 18 & 18 & 17 & 17 & - & 17 & 17 \\
ND & 4 & 4 & 3 & 3 & 3 & 3 & 4 \\
B & 4 & 4 & 4 & 4 & - & 3 & 4 \\
Gap & 0 & 0 & 0 & 0 & - & 0 & $60.46 \%$ \\
Time & 10 & 170 & 81 & 6 & - & 61 & 99,000 \\
CTime & 10 & 180 & 261 & 267 & - & 322 & 99,000 \\
\hline
\end{tabular}

${ }^{\dagger}$ Objective function value not proven to be optimal.

despite their long processing times, and the computational efficiency of the proposed solution strategy could thus be verified once again. While the total processing times for considering all steps were 1,234 seconds and 267 seconds, for cases C41 and C41S, respectively, the complete model was run for 99,000 seconds for both cases, and gaps exceeding $60 \%$ were obtained. The servicing policies can be compared. If four berths are available, the option to service the mobile units and permanent units together is better than segregating them. The costs would be $2.1 \%$ lower in this case. This analysis is valid for the instances presented and might diverge in other cases.

Berth allocation for case C41 can be seen in Figure 6. On the left, the berth number $(\mathrm{B})$ and the departing position number $(\mathrm{P})$ are indicated. On the top, each column represents one day, and the solutions with four, three 
and two berths are compared. The numbers indicated in the colored spaces are the route number for each berth-position and day. The color indicates the type of vessel that was used: red for large size, yellow for mid-size and black for small size.

\begin{tabular}{|c|c|c|c|c|c|c|c|c|c|c|c|c|c|c|c|c|c|c|c|c|c|c|}
\hline \multicolumn{9}{|c|}{ 4B } & \multicolumn{10}{|c|}{ 3B } & \multicolumn{10}{|c|}{ 2B } \\
\hline $\mathrm{B}$ & $\mathrm{P}$ & 1 & 2 & 3 & 4 & 5 & 6 & 7 & 1 & 2 & 3 & 4 & 5 & 6 & 7 & 1 & 2 & 3 & 4 & 5 & 6 & 7 \\
\hline 1 & 1 & & 1094 & & & 1336 & 686 & & 933 & 267 & 1238 & 167 & & 1131 & 113 & 686 & 1131 & 267 & 113 & 265 & 1013 & 1131 \\
\hline 1 & 2 & & & & & & & & 22 & & & 937 & & & & & & 39 & 937 & & & \\
\hline 2 & 1 & 972 & & 933 & 267 & 49 & 167 & & & 1013 & 1336 & 686 & 1331 & 265 & 1094 & 167 & 972 & 1238 & 1336 & 31 & 933 & 49 \\
\hline 2 & 2 & & & & & & & & & & & & & 39 & & 22 & & & & & 1362 & 1094 \\
\hline 3 & 1 & 265 & 113 & 22 & & & 937 & & 1362 & & 49 & 31 & & 972 & & & & & & & & \\
\hline 3 & 2 & & & & & & & & & & & & & & & & & & & & & \\
\hline 4 & 1 & 1131 & & 1331 & 1013 & 1238 & 31 & 1362 & & & & & & & & & & & & & & \\
\hline 4 & 2 & 39 & & & & & & & & & & & & & & & & & & & & \\
\hline
\end{tabular}

Figure 6: Berth allocation for case C41.

In case C41, the results for three berths were the same as for four berths both in terms of fleet composition and in terms of routing costs. Given that the maximum number of departures is four and the number of available berths is three, the routes could be rearranged on different departing days, as indicated in the middle section of Figure 6 . When instance $\mathrm{C} 41$ was processed with two berths, a different fleet composition was obtained. The positive gap of $3.1 \%$ indicates a lower bound equal to 261.82 , which is greater than the objective function value of S4 with three or four berths. Thus, a greater fleet is necessary to allow more departures throughout the planning horizon.

The departure times for case C41 can be seen in Figure 7. The importance of allowing flexibility regarding the departure times (i.e. to allow a departure to take place by $12 \mathrm{pm}$ of the following day) was noticed in the case $\mathrm{C} 41$. This actually happened on $4 \mathrm{~B}$ day $1,3 \mathrm{~B}$ day 6 , and $2 \mathrm{~B}$ day 3 . In case $3 \mathrm{~B}$, for example, there is a departure scheduled to take place on day 6 which actually happens at instant 6.2 (day 7). If no tolerance in the departure time is allowed, one extra large vessel would be needed in place of a mid-size vessel, incurring a cost increase of $2 \%$ (around 5 MM USD). This reinforces that finding a feasible berth allocation with the minimum fleet configuration was made possible due to such a tolerance in the departure time.

When a vessel is assigned to a route, it can begin loading at the very beginning of the scheduled departure day; or it may have to wait for a vessel from a previous day to release the berth; or it may happen that a vessel is the second to occupy a berth on a given day. The waiting time before 


\begin{tabular}{|c|c|c|c|c|c|c|c|c|c|c|c|c|c|c|c|c|c|c|c|c|c|c|}
\hline \multicolumn{1}{|c|}{} & \multicolumn{10}{|c|}{ 4B } & \multicolumn{10}{|c|}{ 2B } \\
\hline $\mathrm{B}$ & $\mathrm{P}$ & 1 & 2 & 3 & 4 & 5 & 6 & 7 & 1 & 2 & 3 & 4 & 5 & 6 & 7 & 1 & 2 & 3 & 4 & 5 & 6 & 7 \\
\hline 1 & 1 & & 1.68 & & & 4.69 & 5.73 & & 0.33 & 1.41 & 2.74 & 3.48 & & 5.75 & 6.47 & 0.73 & 1.75 & 2.41 & 3.57 & 4.33 & 5.69 & 6.75 \\
\hline 1 & 2 & & & & & & & & 0.79 & & & 3.79 & & & & & & 3.10 & 3.88 & & & \\
\hline 2 & 1 & 0.45 & & 2.33 & 3.41 & 4.21 & 5.48 & & & 1.69 & 2.69 & 3.73 & 4.68 & 5.33 & 6.70 & 0.48 & 1.45 & 2.74 & 3.69 & 4.56 & 5.33 & 6.21 \\
\hline 2 & 2 & & & & & & & & & & & & & 6.2 & & 0.95 & & & & & 5.94 & 6.89 \\
\hline 3 & 1 & 0.33 & 1.47 & 2.47 & & & 5.31 & & 0.62 & & 2.21 & 3.56 & & 5.45 & & & & & & & & \\
\hline 3 & 2 & & & & & & & & & & & & & & & & & & & & & \\
\hline 4 & 1 & 0.75 & & 2.68 & 3.69 & 4.74 & 5.56 & 6.62 & & & & & & & & & & & & & & \\
\hline 4 & 2 & 1.43 & & & & & & & & & & & & & & & & & & & & \\
\hline
\end{tabular}

Figure 7: Departure instants for each berth-position for case C41.

starting to load a vessel, added to the loading time and to the time at sea, indicates the vessel cycle time. The rounded value of the cycle time to its upper integer value indicates the number of $c_{v b p l_{1} l_{2}}$ binary variables set to 1 , which allows for computing the maximum number of vessels in use - refer to constraints (12) to (17). Figure 8 indicates the cycle time for all departures for case $\mathrm{C} 41$. For example, for $3 \mathrm{~B}$ day 6 , route 39 starts loading at instant 5.33 , which is the time the previous vessel left the berth (see Figure 7 ). The loading time is 0.87 , and the sailing time added to the trans-shipment time at sea is 2.29 . The cycle time is given by $0.33+0.87+2.29=3.49$, thus requiring the vessel to be in use for four days.

\begin{tabular}{|c|c|c|c|c|c|c|c|c|c|c|c|c|c|c|c|c|c|c|c|c|c|c|}
\hline \multicolumn{1}{|c|}{} & \multicolumn{10}{|c|}{ 4B } & \multicolumn{10}{|c|}{ 2B } \\
\hline $\mathrm{B}$ & $\mathrm{P}$ & 1 & 2 & 3 & 4 & 5 & 6 & 7 & 1 & 2 & 3 & 4 & 5 & 6 & 7 & 1 & 2 & 3 & 4 & 5 & 6 & 7 \\
\hline 1 & 1 & & 2.76 & & & 2.91 & 2.94 & & 1.53 & 1.85 & 2.98 & 1.10 & & 2.97 & 1.89 & 2.94 & 2.97 & 1.85 & 1.98 & 1.56 & 2.82 & 2.97 \\
\hline 1 & 2 & & & & & & & & 2.71 & & & 1.89 & & & & & & 3.56 & 1.98 & & & \\
\hline 2 & 1 & 1.89 & & 1.53 & 1.85 & 0.97 & 1.10 & & & 2.82 & 2.91 & 2.94 & 2.87 & 1.56 & 2.78 & 1.10 & 1.89 & 2.98 & 2.91 & 2.71 & 1.53 & 0.97 \\
\hline 2 & 2 & & & & & & & & & & & & & 3.49 & & 2.86 & & & & & 2.99 & 2.97 \\
\hline 3 & 1 & 1.56 & 1.89 & 2.38 & & & 1.41 & & 2.67 & & 0.97 & 2.71 & & 1.89 & & & & & & & & \\
\hline 3 & 2 & & & & & & & & & & & & & & & & & & & & & \\
\hline 4 & 1 & 2.97 & & 2.87 & 2.82 & 2.98 & 2.71 & 2.67 & & & & & & & & & & & & & & \\
\hline 4 & 2 & 3.90 & & & & & & & & & & & & & & & & & & & & \\
\hline
\end{tabular}

Figure 8: Cycle times for each berth-position for case C41.

According to Table 4, in case C41 the fleet is composed of seven vessels. Figures 9 to 11 present possible arrangements for the vessels' assignments depending on the number of berths. In the figures, the number of berths is indicated in the first line, and the days are indicated in the second line. Then, each additional line corresponds to a vessel, indicated by its class and its number. For each vessel, the rectangles are as large as the cycle time of each route, whose number is indicated inside the rectangle. The colors 
indicate the vessel classes: red for large size, yellow for mid-size and black for small size. The light and dark colors are meant to highlight the routes' assignments over each week. As one may notice, the routes selected for the large-size vessels cannot be repeated for the same vessel in two consecutive weeks. If the vessels were required to perform the same routes from one period to the other the proposed solution would not be feasible. However, by considering the vessels as belonging to a pool of available resources, more sophisticated and cost-effective solutions can be built.

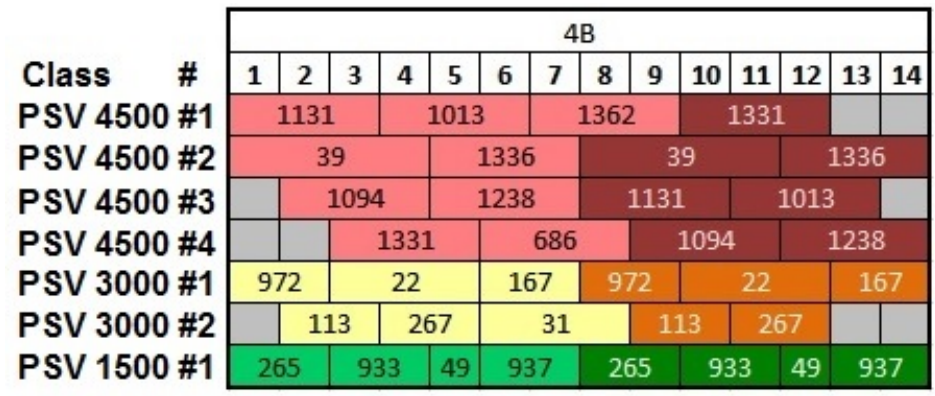

Figure 9: Vessels' allocation for case C41 (four berths).

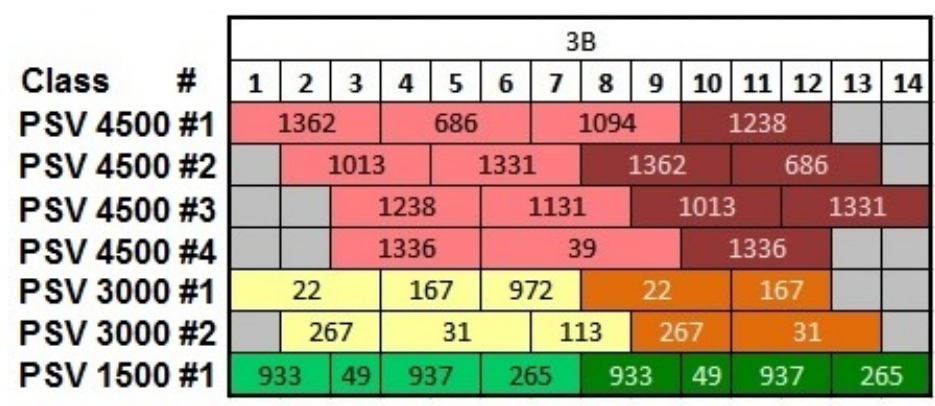

Figure 10: Vessels' allocation for case C41 (three berths). 


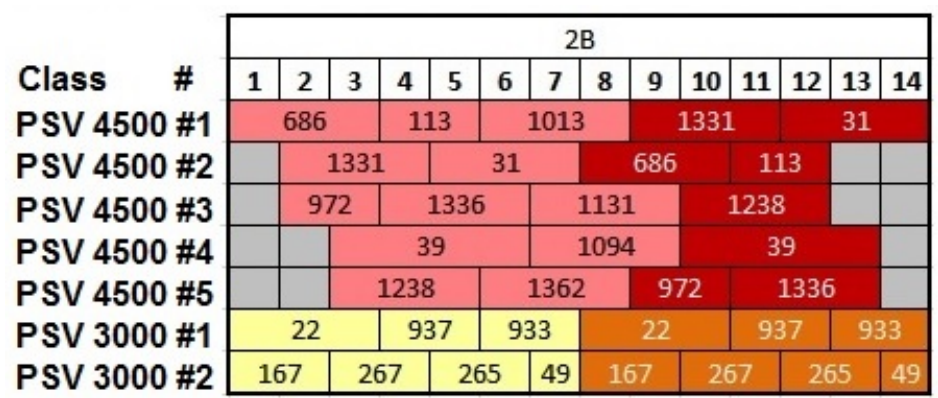

Figure 11: Vessels' allocation for case C41 (two berths).

\subsection{Results for Cases C79 and CryS}

Cases C79 and C79S were also tested and the results are presented in Table 5. In case C79 with four berths, Steps 1 to 3 did not present any variation related to the fleet composition. However, in Step 4, the solution from Step 3 could not be confirmed, and a mid-sized vessel was added to the fleet. The gap was $5.58 \%$ with no progress of the lower bound throughout the whole processing time. Step 4 was also tested for five and six berths (5B and $6 \mathrm{~B}$ ) and only with six berths the Step 3 solution could be scheduled with the same fleet. In case C79S with four berths, the model processing in Step 1 and in Step 2 were not able to close the gap, which was $0.56 \%$ after 99,000 seconds. The Step 1 solution was obtained after 1,549 seconds, and the gap was $2.04 \%$. Then it took almost 97,500 seconds to reduce the gap to $0.56 \%$. In Step 2, the final solution was generated at instant 5,160 with a gap equal to $0.56 \%$. The Step 3 model was interrupted after 61,180 seconds due to an out-of-memory error without having generated any primal solution.

We also tested how Steps 2, 3 and 4 would perform if the upper bound of a previous solution step was informed in constraints (36), (39), (43) and (44) instead of the lower bound. This is different from the previous cases, where the initial steps always produced optimal solutions, and thus the lower bounds and the upper bounds were the same. These new tests are registered in the columns marked with an asterisk. It was observed that this procedure produced rapid convergence in the model and very good-quality solutions could be achieved, although we cannot claim to have obtained the optimal solution. It was possible to find a solution serving 79 offshore units using only four berths and the solution obtained in Step 4 has a gap of only $0.56 \%$ (the same as in the Step 1 solution). Therefore, we consider this alternative 
Table 5: Results for cases C79 and C79S

\begin{tabular}{|l|c|c|c|c|c|c|c|}
\hline C79 & S1 & S2 & S3 & S4 & S4(5B) & S4(6B) & CM \\
\hline OF & 527 & 527 & 558.55 & $591.55^{\dagger}$ & $591.55^{\dagger}$ & 558.55 & - \\
VC & 527 & 527 & 527 & 560 & 560 & 527 & - \\
RC & - & - & 31.55 & 31.55 & 31.55 & 31.55 & - \\
V0 & 8 & 8 & 8 & 8 & 8 & 8 & - \\
V1 & 6 & 6 & 6 & 7 & 7 & 6 & - \\
V2 & 1 & 1 & 1 & 1 & 1 & 1 & - \\
NR & 38 & 38 & 38 & 38 & 38 & 38 & - \\
ND & 8 & 8 & 8 & 7 & 6 & 7 & - \\
B & 4 & 4 & 4 & 4 & 5 & 6 & 4 \\
Gap & 0 & 0 & 0 & $5.58 \%$ & $5.58 \%$ & 0 & - \\
Time & 3,274 & 5,755 & 36,270 & 99,000 & 99,000 & 64,803 & 99,000 \\
CTime & 3,274 & 9,029 & 45,299 & 144,299 & 144,299 & 110,102 & 99,000 \\
\hline \hline C79S & S1 & S2 & S3 & S2* & S3* & S4(4B)* & CM \\
\hline OF & $540^{\dagger}$ & $540^{\dagger}$ & $570.46^{\dagger}$ & 540 & 570.37 & 570.37 & - \\
VC & 540 & 540 & - & 540 & 540 & 540 & - \\
RC & - & - & - & - & 30.37 & 30.37 & - \\
V0 & 9 & 9 & - & 9 & 9 & 9 & - \\
V1 & 6 & 6 & - & 6 & 6 & 6 & - \\
V2 & 0 & 0 & - & 0 & 0 & 0 & - \\
NR & 36 & 35 & - & 35 & 34 & 34 & - \\
ND & 8 & 6 & - & 6 & 6 & 6 & - \\
B & 4 & 4 & 4 & 4 & 4 & 4 & 4 \\
Gap & $0.56 \%$ & $0.56 \%$ & $0.49 \%$ & 0 & 0 & 0 & - \\
Time & 99,000 & 99,000 & 61,180 & 161 & 1,771 & 8,653 & 99,000 \\
CTime & 99,000 & 198,000 & 259,180 & 99,161 & 100,932 & 109,585 & 99,000 \\
\hline
\end{tabular}

$\dagger$ Objective function value not proven to be optimal.

* Upper bound of a previous solution step informed instead of the lower bound.

as a valid and useful solution strategy that could be used in practice. The complete model was tested for both cases to assess the effectiveness of the proposed methodology. In both cases, no feasible solution was obtained after 99,000 seconds.

\subsection{Proposed Solution Strategy Performance}

The comparison between complete mathematical model $(\mathrm{CM})$ with the proposed solution strategy (PSS) considering the overall results from Step 1 to Step 4 is presented in Table 6. Based on the presented results, one may conclude that the proposed methodology is capable of significantly reducing the computational time and to provide an optimum solution for most of the cases considered. For case C10 both PSS and CM achieved the optimum 
solution and the PSS processing time is significantly lower than the CM processing time. For cases C15, C41 and C41S the PSS was able to achieve the optimum solution and the CM could not. As one might notice, the PPS spent a significant reduced amount of time to generate optimal solution. For the cases with 79 offshore units, in C79 a good solution has been found by the PPS but no solution has been found by the CM. For case C79S both PPS and the CM struggle to find a feasible solution. A large processing time is obtained for a few cases, particularity for cases C79 and C79S. For this kind of problem, a large processing time of thousands of seconds is not an issue, once it has to be solved around every quarter of a year or more.

Table 6: Comparison between the proposed solution strategy and the complete model

\begin{tabular}{|l|c|c||l|c|c|}
\hline C10 & PSS & CM & C15 & PSS & CM \\
\hline OF & 53.77 & 53.77 & OF & 80.37 & $89.43^{\dagger}$ \\
Gap & 0 & 0 & Gap & 0 & $57.90 \%$ \\
Time & 115 & 84,340 & Time & 6,812 & 21,289 \\
\hline \hline C41 & PSS & CM & C41S & PSS & CM \\
\hline OF & 256.59 & $289.23^{\dagger}$ & OF & 261.99 & $305.00^{\dagger}$ \\
Gap & 0 & $62.60 \%$ & Gap & 0 & $60.46 \%$ \\
Time & 487 & 99,000 & Time & 267 & 99,000 \\
\hline \hline C79 & PPS & CM & C79S & PPS & CM \\
\hline OF & $591.55^{\dagger}$ & - & OF & - & - \\
Gap & $5.58 \%$ & - & Gap & - & - \\
Time & 144,299 & 99,000 & Time & - & - \\
\hline \hline
\end{tabular}

$\dagger$ Objective function value not proven to be optimal.

An assessment to further investigate the effectiveness of breaking down the complete model into steps has been performed for cases C15, C41 and C79. The results are presented in Table 7 and the following denomination has been used in the columns: i) S1-3 is the consolidated result for steps 1 to 3 ; ii) S3' is the result for the Step 3 model without the lower-bound constraint (39) and without using the Step 2 solution as an initial solution; iii) S3" is the result for Step 3 without the use of the Step 2 solution as an initial solution; iv) S3 is the result for Step 3 (previously calculated); v) S4" is the result of Step 4 without the use of the Step 3 solution as a constraint, i.e. without considering constraints (41) and (42); vi) S4 is the result for Step 4 (previously calculated).

If one compares S1-3 to S3' it is possible to verify the effectiveness of breaking down the Step 3 model in Step 1 and Step 2. For cases C41 and 
Table 7: Performance assessment of Step 3 and Step 4

\begin{tabular}{|l||c|c||c|c||c|c|}
\hline C15 & S1-3 & S3' & S3" & S3 & S4" & S4 \\
\hline Time & 6,806 & 3,251 & 438 & 496 & 13,006 & 6 \\
\hline OF & 80.37 & 80.37 & 80.37 & 80.37 & 80.37 & 80.37 \\
\hline GAP & 0 & 0 & 0 & 0 & 0 & 0 \\
\hline \hline C41 & S1-3 & S3' & S3" & S3 & S4" & S4 \\
\hline Time & 140 & 394 & 122 & 77 & 99,000 & 348 \\
\hline OF & 256.59 & 256.59 & 256.59 & 256.59 & $294.95^{\dagger}$ & 256.59 \\
\hline GAP & 0 & 0 & 0 & 0 & $13.00 \%$ & 0 \\
\hline \hline C79 & S1-3 & S3' & S3" & S3 & S4" & S4 \\
\hline Time & 42,685 & 95,192 & 99,000 & 36,270 & 99,000 & 99,000 \\
\hline OF & 558.55 & 558.55 & $558.55^{\dagger}$ & 558.55 & $705.24^{\dagger}$ & $591.55^{\dagger}$ \\
\hline GAP & 0 & 0 & $0.02 \%$ & 0 & $20.80 \%$ & $5.58 \%$ \\
\hline
\end{tabular}

$\dagger$ Objective function value not proven to be optimal.

$\mathrm{C} 79$, the reduction on the processing time is clearly demonstrated. However, as one might notice in case C15 it is more efficient to run Step 3 without Step 1 and 2, and the use of the lower bound and of the initial solution has not been demonstrated as advantageous for Step 3 model. If one compares S3" to $\mathrm{S} 3$ it is possible to verify the effectiveness of using the Step 2 solution in Step 3, as for cases $\mathrm{C} 41$ and $\mathrm{C} 79$ the processing time has been significantly reduced. For case $\mathrm{C} 15$, the processing time remains almost the same. If one compares S4" to S4 it is possible to verify the effectiveness of using the routes obtained in Step 3 as constraints (41) and (42). For cases C15 and $\mathrm{C} 41$, there was a significant reduction in the processing time (by a thousand times less). For case C79, neither S4 nor S4" has closed the gap to zero; however a better solution could be provided by using the Step 3 solution as an input to Step 4.

This assessment demonstrates that the effectiveness of breaking down the model into steps (1 to 3 ) is not always the most efficient manner to solve the problem, although it was efficient for some cases. This assessment also demonstrates the effectiveness of using Step 3 routes as the initial solution to Step 4 for all evaluated instances. The overall evaluation of the method presented in Table 6 demonstrates the overall effectiveness of breaking down the model into steps for all instances. 


\section{Concluding Remarks}

In this paper we have investigated the integration of berth allocation decisions to the fleet composition and periodic routing problem. This is a real complex and hard-to-solve problem found in some oil and gas industries, such as in the Brazilian case, where the supply operation is uninterrupted, both at the onshore base and the offshore units. The integration of berth allocation decisions extends previous contributions on the planning of platform supply vessels, where the number of vessels that can be loaded on each day was limited by the number of available berths. In our case, the berth scheduling problem is considered together with the periodic routing problem. Also, in order to model the fleet size, a pool of vessels was considered, one for each class, and this modeling strategy seemed to be effective.

In this paper we presented a solution strategy scheme to solve real instances, as the complete mathematical model is hard to solve due to its combinatorial nature and many constraints. This strategy consists of sequentially solving relaxed versions of the problem, adding more complexity with each step. The proposed strategy consisted of forcing the routes obtained in Step 3 to be scheduled in the fourth step, which could not be feasible from the berth-scheduling perspective. To deal with this issue, the Step 4 model allowed the modification of the departing days, by selecting any valid departure pattern, by considering the use of vessels from different classes, and by allowing late departures up to given tolerance. Although this solution approach may hinder the achieving of the optimal solution, from the practical point of view, good-quality solutions were obtained. Instances were solved based on a real case from a Brazilian oil and gas company, including a case with up to 79 offshore units grouped into clusters.

Future research topics include the development of heuristic methods, and the consideration of stochastic demand and stochastic travel times.

\section{Acknowledgements}

The authors would like to thank the anonymous reviewers for their valuable comments that greatly contributed to improving the final version of the paper. This work was partially supported by the National Council for Sci-

entific and Technological Development - CNPq, under grant 305180/2016-9. 


\section{References}

Aas, B., Gribkovskaia, I., Halskau-Sr, Ø., Shlopak, A., 2007. Routing of supply vessels to petroleum installations. International Journal of Physical Distribution \& Logistics Management 37 (2), 164-179.

Aas, B., Halskau-Sr, Ø., Wallace, S. W., 2009. The role of supply vessels in offshore logistics. Maritime Economics \& Logistics 11 (3), 302-325.

Agra, A., Oliveira, M., 2018. Mip approaches for the integrated berth allocation and quay crane assignment and scheduling problem. European Journal of Operational Research 264 (1), 138-148.

Baptista, S., Oliveira, R. C., Zúquete, E., 2002. A period vehicle routing case study. European Journal of Operational Research 139 (2), 220-229.

Bierwirth, C., Meisel, F., 2015. A follow-up survey of berth allocation and quay crane scheduling problems in container terminals. European Journal of Operational Research 244 (3), 675-689.

Christofides, N., Beasley, J. E., 1984. The period routing problem. Networks 14 (2), 237-256.

Cordeau, J.-F., Laporte, G., Legato, P., Moccia, L., 2005. Models and tabu search heuristics for the berth-allocation problem. Transportation Science 39 (4), 526-538.

Cuesta, E. F., Andersson, H., Fagerholt, K., Laporte, G., 2017. Vessel routing with pickups and deliveries: An application to the supply of offshore oil platforms. Computers \& Operations Research 79, 140-147.

Døsen, H. C., Langeland, J., 2015. Offshore freight rate determinants. Master's thesis, Norwegian School of Economics, Bergen/NO.

Eskandari, H., Gribkovskaia, I., Barceló, J., 2016. A simulation-based multiobjective optimization study of the fleet sizing problem in the offshore industry. Maritime Economics \& Logistics 18 (4), 436-457.

Fagerholt, K., Lindstad, H., 2000. Optimal policies for maintaining a supply service in the norwegian sea. Omega 28 (3), 269-275. 
Francis, P. M., Smilowitz, K. R., Tzur, M., 2008. The period vehicle routing problem and its extensions. In: Golden, B., Raghavan, S., Wasil, E. (Eds.), The Vehicle Routing Problem: Latest Advances and New Challenges. Vol. 43 of Operations Research/Computer Science Interfaces Series. Springer, Boston, pp. 73-102.

Gribkovskaia, I., Laporte, G., Shlopak, A., 2008. A tabu search heuristic for a routing problem arising in servicing of offshore oil and gas platforms. The Journal of the Operational Research Society 59 (11), 1449-1459.

Halvorsen-Weare, E. E., Fagerholt, K., 2017. Optimization in offshore supply vessel planning. Optimization and Engineering 18 (1), 317-341.

Halvorsen-Weare, E. E., Fagerholt, K., Nonås, L. M., Asbjørnslett, B. E., 2012. Optimal fleet composition and periodic routing of offshore supply vessels. European Journal of Operational Research 223 (2), 508-517.

Hemmelmayr, V. C., 2015. Sequential and parallel large neighborhood search algorithms for the periodic location routing problem. European Journal of Operational Research 243 (1), 52-60.

Imai, A., Nagaiwa, K. I., Tat, C. W., 1997. Efficient planning of berth allocation for container terminals in asia. Journal of Advanced Transportation 31 (1), 75-94.

Imai, A., Nishimura, E., Papadimitriou, S., 2001. The dynamic berth allocation problem for a container port. Transportation Research Part B: Methodological 35 (4), 401-417.

Kisialiou, Y., Gribkovskaia, G., Laporte, G., 2018. The periodic supply vessel planning problem with flexible departure times and coupled vessels. Computers and Operations Research 94, 52-64.

Koç, Ç., 2016. A unified-adaptive large neighborhood search metaheuristic for periodic location-routing problems. Transportation Research Part C: Emerging Technologies 68, 265-284.

Lim, A., 1998. The berth planning problem. Operations Research Letters $22(2-3), 105-110$. 
Maisiuk, Y., Gribkovskaia, I., 2014. Fleet sizing for offshore supply vessels with stochastic sailing and service times. Procedia Computer Science 31, 939-948.

Pratap, S., Nayak, A., Kumar, A., Cheikhrouhou, N., Tiwari, M. K., 2017. An integrated decision support system for berth and ship unloader allocation in bulk material handling port. Computers \& Industrial Engineering 96, 386-399.

Prodon, C., 2011. A hybrid evolutionary algorithm for the periodic location routing problem. European Journal of Operational Research 210 (2), 204212.

Ribeiro, G. M., Mauri, G. R., Beluco, S. C., Lorena, L. A. N., Laporte, G., 2016. Berth allocation in an ore terminal with demurrage, despatch and maintenance. Computers \& Industrial Engineering 96, 8-15.

Seixas, M. P., Mendes, A. B., Barretto, M. R. P., da Cunha, C. B., Brinati, M. A., Cruz, R. E., Yue, W., Wilson, P. A., 2016. A heuristic approach to stowing general cargo into platform supply vessels. Journal of the Operational Research Society 67 (1), 148-158.

Shyshou, A., Gribkovskaia, I., Barceló, J., 2010. A simulation study of the fleet sizing problem arising in offshore anchor handling operations. European Journal of Operational Research 203 (1), 230-240.

Shyshou, A., Gribkovskaia, I., Laporte, G., Fagerholt, K., 2012. A large neighbourhood search heuristic for a periodic supply vessel planning problem arising in offshore oil and gas operations. INFOR: Information Systems and Operational Research 50 (4), 195-204.

Stålhane, M., Vefsnmo, H., Halvorsen-Weare, H. H., Hvattum, L. M., Nonås, L. M., 2016. Vessel fleet optimization for maintenance operations at offshore wind farms under uncertainty. Energy Procedia 94, 357-366. 